\title{
Stem cell culture shock
}

Available human embryonic stem cell (hESC) lines have incorporated substantial amounts of an immunogenic molecule of animal origin - amounts likely to cause immune rejection in humans. The sources of contamination are the bovine and murine products currently required to grow undifferentiated $\mathrm{hESCs}$. This discovery (Martin et al., Nature Medicine 11, 228-232; 2005) has been picked up by the press largely as a call for the development of new hESC lines. There are a number of other reasons why more hESC lines will be needed before stem cell therapy finally reaches the clinic, but what this finding also highlights is a pressing need for improved culture systems to grow these cells in the absence of animal products.

Although reagents of animal origin have been used in the manufacturing of biological therapeutics such as vaccines, they are the subject of serious safety concerns. In addition to the potential for causing hypersensitivity reactions in patients, the most critical issue is the risk that animal components may introduce pathogens. Therapeutic products can be tested for the presence of a subset of infectious agents, but not all of them. In an attempt to detect these so-called adventitious agents, crude toxicity tests are also commonly performed involving injection in mice, hen eggs and guinea pigs, but the sensitivity of these tests is questionable.

Furthermore, let's not forget that when we talk about hESC therapy we are projecting a considerable number of years in the future. Meanwhile, new animal pathogens could emerge or escape containment. To cite a precedent, fetal bovine serum has only come under increased scrutiny for contamination with the causative agent of bovine spongiform encephalopathy (BSE) since the outbreak of the BSE epidemic in the United Kingdom. In addition, there is still no reliable test to detect the BSE infectious agent, and bovine sera are approved for manufacturing of biological therapeutics on the basis of country of origin. This measure now works reasonably well, and suppliers rely on North American sources, which have remained largely untouched by the disease. Nevertheless, an outbreak of BSE in American herds, a possibility that cannot be ruled out, would considerably complicate the situation.

Further complicating the detection of adventitious agents is the fact that stem cell therapy will most likely never be a 'shelf product'. It will either be autologous, done by modification of the patient's own cells, or allogenic, done by modification of cells from a human leukocyte antigen (HLA)-matched source. As such, each patient will receive a different product. Ideally each batch of therapeutic product should undergo extensive safety testing, but in practice, testing may be restricted by the time-sensitive nature or paucity of the material.

Thus, even if a manufacturing process that involves products of animal origin and of undefined composition is not an absolute show stopper for the initiation of clinical trials, it is not a long-term option for sustainable therapies. hESCs have shown promise for combating frequently occurring disorders such as diabetes and Alzheimer disease. At such large scales, monitoring the quality of each batch of uncertain reagent can be expected to further drive up the cost of an already complex approach, limiting patients' access to therapy.

In addition, if an adverse event related to one of the culture components were to occur during a clinical trial, the entire stem cell field could suffer a major setback, as the experience of the gene therapy community has shown. With so much debate already going on, another controversy is just what the stem cell field needs to avoid, especially given its increasing dependence on public funding.

Now is the time to act on these practical issues. As researchers are focusing on stem cells' biology and ways to exploit their therapeutic potential, let's not forget the logistical issues that will arise when researchers finally get ready to inject a patient. Given the complexity of the problem, there is no magic bullet to expect. The solution will most likely come as the sum of incremental steps, partly as the result of increasing knowledge of stem cell biology and partly from empirical approaches. The good news is that a number of efforts are already ongoing. The report by Thomson and colleagues in this issue (p. 185) is one example of the successful replacement of some of the mouse-derived products by an appropriate dosage of two signalingpathway effectors. With the appropriate focus, such efforts should ultimately reveal a recipe for growing $\mathrm{hESC}$ using only chemically characterized reagents.

Hopefully, by the time researchers have developed ways of turning stem cells into differentiated cells of therapeutic interest, the field will be ready to make the transition from bench to bedside and produce these promising therapeutics under safe and costeffective conditions. 